\title{
Exercise counseling of primary care physicians in metabolic syndrome and cardiovascular diseases is associated with their specialty and exercise habits
}

\author{
This article was published in the following Dove Press journal: \\ International Journal of General Medicine \\ 17 June 2014 \\ Number of times this article has been viewed
}

\section{Yoshiyuki Morishita' \\ Atushi Miki' \\ Mari Okada' \\ Satoshi Tsuboi ${ }^{2}$ \\ Kenichi Ishibashi ${ }^{3}$ \\ Yasuhiro Ando' \\ Daisuke Nagata' \\ Eiji Kusano ${ }^{4}$}

'Division of Nephrology,

Department of Medicine, Jichi Medical University, Tochigi, Japan;

${ }^{2}$ Department of Public Health, Jichi Medical University, Tochigi, Japan;

${ }^{3}$ Department of Medical Physiology, Meiji Pharmaceutical University,

Tokyo, Japan; ${ }^{4}$ Utsunomiya Social Insurance Hospital, Tochigi, Japan
Correspondence: Yoshiyuki Morishita Division of Nephrology, Department of Medicine, Jichi Medical University, 33 II-I, Yakushiji, Shimotsuke-city, Tochigi 329-0498, Japan

Tel +8I 285587346

Fax +8I 285444869

Email ymori@jichi.ac.jp
Background: We investigated the practice of exercise counseling of primary care physicians in metabolic syndromes and cardiovascular diseases and its association with their age class, specialty, work place, and their own exercise habits.

Subjects and methods: The subjects were 3,310 medical doctors who had graduated from Jichi Medical University in Japan. The study instrument was a self-administered questionnaire to investigate their age class, specialty, workplace, exercise habits, and exercise counseling for their patients.

Results: Overall, 839 completed responses were analyzed from a total of 933 that were received $(28.2 \%)$. The primary care physicians whose specialties were internal medicine and general medicine significantly more often recommended exercise in diabetes mellitus, hyperlipidemia, heart failure, and hypertension cases than those whose specialties were surgery and pediatrics. The primary care physicians whose specialty was pediatrics recommended exercise less often in apoplexia cases than those whose specialties were internal medicine, general medicine, and surgery. Their exercise habits were positively associated with their recommendation of exercise in hyperlipidemia, heart failure, and hypertension cases; however, these associations were not observed in diabetes mellitus and apoplexia. The primary care physicians' age class and work place showed no association with their exercise recommendations in metabolic syndrome and cardiovascular diseases.

Conclusion: The primary care physicians whose specialties were internal medicine and general medicine significantly more often recommended exercise in diabetes mellitus, hyperlipidemia, heart failure, and hypertension cases. In addition, their own exercise habits were positively associated with their recommendation of exercise in hyperlipidemia, heart failure, and hypertension cases.

Keywords: primary care physician, self-administered questionnaire, exercise recommendation, metabolic syndrome, cardiovascular diseases

\section{Introduction}

Exercise counseling is very important for the prevention and inhibition of the progression of many chronic diseases, including metabolic syndrome and cardiovascular diseases. ${ }^{1-6}$ Increased physical activity has been reported to have beneficial effects on the incidence and prognosis of these chronic diseases, as well as overall morbidity and mortality. ${ }^{1-6}$ Primary care physicians have important roles in exercise counseling for these patients because they provide them with close and continuous care. A few studies reported primary care physicians' exercise counseling in cases of chronic disease, and suggested that their lifestyle may be associated with their exercise counseling for 
such cases. ${ }^{7-11}$ However, primary care physicians' exercise counseling in cases of metabolic syndrome and cardiovascular diseases has not been fully reported. Therefore, we conducted a cross-sectional study to investigate the practice of exercise counseling of primary care physicians in cases of chronic diseases such as metabolic syndrome, cardiovascular diseases, and chronic kidney disease. We previously reported that primary care physicians offering exercise counseling for chronic kidney disease patients was positively associated with their own exercise habits. ${ }^{12}$ In the present study, we performed a subanalysis of our survey to investigate the practice of primary care physicians' exercise counseling for cases of metabolic syndrome (diabetes mellitus and hyperlipidemia) and cardiovascular diseases (apoplexia, heart failure, and hypertension), and its association with their age class, specialty, work place, and own exercise habits.

\section{Subjects and methods}

This study was conducted in accordance with the Declaration of Helsinki and was approved by the ethics committee of Jichi Medical University.

\section{Subjects}

The subjects of this cross-sectional study were 3,310 medical doctors who graduated from Jichi Medical University in Japan from 1978 to 2012. The majority of graduates of this university have been working as primary care physicians.

\section{Study instrument}

The study instrument was a self-administered questionnaire that was mailed along with an addressed and stamped envelope for reply. It was designed to investigate the primary care physicians' characteristics (age, specialty, work place, personal exercise frequency and intensity), exercise counseling for cases of metabolic syndrome and cardiovascular diseases, and management of chronic kidney disease. The parameters canvased in the questionnaire of the primary care physicians' characteristics were reported in our previous manuscript ${ }^{13}$ and are also shown in Table 1 . The question on exercise counseling for patients with metabolic syndrome and cardiovascular disease was as follows: diseases for which you recommend exercise (multiple selections allowed): 1) diabetes mellitus; 2) hyperlipidemia; 3) apoplexia; 4) heart failure; 5) arterial hypertension; and 6) others.

\section{Exercise habits}

The exercise volume (metabolic equivalents of task [Mets] $\times$ hours/week) was calculated as representing their exercise
Table I The characteristics of primary care physicians

\begin{tabular}{ll}
\hline & $\mathbf{n}(\%)$ \\
\hline Age & \\
$24-30$ & $71(8.5)$ \\
$30-40$ & $259(30.9)$ \\
$40-50$ & $265(31.6)$ \\
$50-60$ & $230(27.4)$ \\
$\geq 60$ & $14(1.7)$ \\
Total & $839(100)$ \\
Specialty & \\
Internal medicine & $387(46.1)$ \\
Surgery & $158(18.8)$ \\
General medicine & $155(18.5)$ \\
Pediatrics & $44(5.2)$ \\
Others & $95(11.3)$ \\
Total & $839(100)$ \\
Work place & \\
University hospital & $97(I 1.6)$ \\
Polyclinic hospital & $191(22.8)$ \\
Hospital & $236(28.1)$ \\
Clinic & $273(32.5)$ \\
Others & $42(5.0)$ \\
Total & $839(100)$ \\
Exercise frequency & \\
Daily & $104(12.4)$ \\
$\geq 2-3$ times/week & $233(27.8)$ \\
$\geq I$ time/week & $224(26.7)$ \\
I I time/month & $278(33.1)$ \\
Total & $839(100)$ \\
Exercise intensity & \\
High ( $\geq 6$ Mets) & $264(29.5)$ \\
Moderate (4-6 Mets) & $199(22.2)$ \\
Mild (3-4 Mets) & $295(32.9)$ \\
Very mild (<3 Mets) & $68(7.6)$ \\
Total & $839(100)$ \\
\hline
\end{tabular}

habit by multiplying their exercise frequency by their exercise intensity. The details of this calculation were reported in our previous manuscript, ${ }^{13}$ but a brief explanation is provided here. Their exercise frequency was scored as follows: daily was given a score of 3.5 ( 7 days $\times 0.5$ hours), $\geq 2-3$ times/week was 1.25 ( 2.5 days $\times 0.5$ hours), $\geq 1$ time/week was 0.5 ( 1 day $\times 0.5$ hours), and $\leq 1$ time/month was 0.125 ( 0.25 days $\times 0.5$ hours). Their exercise intensity was scored as follows: high was 6 (6 Mets), moderate was 5 (5 Mets), mild was 4 (4 Mets), very mild was 3 (3 Mets), and none was 0 (0 Met).

\section{Statistical analysis}

The associations between primary care physicians' exercise recommendations for patients with metabolic syndrome and cardiovascular diseases and their own age class, specialty, work place, and exercise volume were analyzed by multiple logistic regression analyses. SPSS Statistics version 21 software (IBM, Armonk, NY, USA) was used for the 
statistical analyses. Values of $P<0.01$ were considered to be significant.

\section{Results}

The survey was mailed to 3,310 medical doctors, with responses being received from 933 in total (28.2\%). Of these, 37 responses were excluded from this study due to their inadequacy, such as a blank response for age, specialty, work place, or exercise habits. Responses marked "others" in terms of exercise frequency $(n=52)$ and exercise intensity $(n=6)$ were excluded from the analyses to evaluate the exercise volume because they could not be scored. ${ }^{13}$ The remaining 839 medical doctors' responses to the self-administered questionnaire were analyzed.

\section{The primary care physicians' age class, specialty, work place, and own exercise habits}

The analyzed primary care physicians' age class, specialty, work place, and exercise habits are shown in Table 1. Their exercise volume was calculated (average \pm standard deviation) to be $21 \pm 5.67$ (Mets $\times$ hours/week).

\section{Exercise counseling by primary care physicians for patients with metabolic syndrome and cardiovascular diseases}

The proportions of those recommending exercise among the total primary care physicians for patients with metabolic syndrome and cardiovascular diseases were as follows (number of primary care physicians who recommend exercise/total): diabetes mellitus, 758/839 (90.3\%); hyperlipidemia, 659/839 (78.5\%); apoplexia, 325/839 (38.7\%); heart failure, 150/839 (17.9\%); and arterial hypertension, 517/839 (61.6\%).

\section{The association between the exercise counseling by primary care physicians for patients with metabolic syndrome and cardiovascular diseases and their age class, specialty, work place, and own exercise habits}

Multiple logistic regression analyses showed that the primary care physicians whose specialties were internal medicine and general medicine significantly more often recommended exercise than those whose specialties were surgery and pediatrics, in cases of diabetes mellitus $(P<0.01)$, hyperlipidemia $(P<0.01)$, heart failure $(P<0.01)$, and arterial hypertension $(P<0.01)$ (Table 2$)$. It was also shown that the primary care physicians whose specialty was pediatrics less often recommended exercise in apoplexia cases than those whose specialties were internal medicine, general medicine and surgery $(P<0.01)$ (Table 2). Their own exercise habits were also positively associated with their recommendation of exercise in cases of hyperlipidemia $(P<0.01)$, heart failure $(P<0.01)$, and hypertension $(P<0.01)$; however, these associations were not observed in diabetes mellitus and apoplexia patients (Table 2). Their age class and work place showed no significant association with their exercise counseling (Table 2).

\section{Discussion}

The results in the present study showed that the exercise recommendation level of primary care physicians for patients with metabolic syndrome was high. In contrast, their exercise recommendation level for hypertension cases was moderate, while those for cases of apoplexia and heart failure were low. Furthermore, the primary care physicians whose specialties were internal medicine and general medicine significantly more often recommended exercise than those whose specialties were surgery and pediatrics, for patients with metabolic syndrome and cardiovascular diseases. Their exercise habits were also positively associated with their recommendation of exercise in cases of hyperlipidemia, heart failure, and hypertension; however, their age class and work place showed no significant association with their exercise counseling for patients with metabolic syndrome and cardiovascular diseases.

Exercise counseling is very important in chronic diseases such as metabolic syndrome and cardiovascular diseases because exercise can improve physical capacity and prevent disease progression. ${ }^{1-6}$ Primary care physicians have a pivotal role in terms of exercise counseling for these patients because they manage them at the front line. Several studies reported that patients want to receive physical activity counseling from their primary care physicians. ${ }^{14,15} \mathrm{In}$ addition, it was suggested that counseling by primary care physicians could contribute to improve patients' exercise habits. ${ }^{16}$

Previous studies have also suggested that physicians' own lifestyle may influence their lifestyle counseling for patients. ${ }^{7-11}$ Abramson et al reported that physicians who exercise regularly are more likely to counsel patients on the merits of exercise. ${ }^{9}$ They also reported that pediatricians and geriatricians counsel fewer patients about exercise than family practitioners and internists. ${ }^{9}$ Wells et al also reported 
Table 2 Multivariate logistic regression analyses to investigate the relationships of the diseases for which primary care physicians recommend exercise and their age, specialty, work place, and own exercise volume

\begin{tabular}{|c|c|c|c|c|c|}
\hline & \multicolumn{2}{|c|}{ Exercise recommendation } & \multirow[t]{2}{*}{ OR } & \multirow[t]{2}{*}{$95 \% \mathrm{Cl}$} & \multirow[t]{2}{*}{$P$-value } \\
\hline & (yes) & (no) & & & \\
\hline \multicolumn{6}{|l|}{ Diabetes mellitus } \\
\hline \multicolumn{6}{|l|}{ Age } \\
\hline 24-30 & 67 & 4 & 1 & ref & ref \\
\hline $30-40$ & 241 & 18 & 0.7 & $0.21-2.29$ & 0.55 \\
\hline $40-50$ & 234 & 31 & 0.47 & $0.15-1.51$ & 0.2 \\
\hline $50-60$ & 202 & 28 & 0.4 & $0.12-0.133$ & 0.13 \\
\hline$\geq 60$ & 14 & 0 & $N / A$ & $\mathrm{~N} / \mathrm{A}$ & N/A \\
\hline \multicolumn{6}{|l|}{ Specialty } \\
\hline Internal medicine & 376 & II & I & ref & ref \\
\hline Surgery & 130 & 28 & 0.14 & $0.07-0.31$ & $*<0.01$ \\
\hline General medicine & $|5|$ & 4 & 0.97 & $0.30-3.11$ & 0.95 \\
\hline Pediatrics & 30 & 14 & 0.06 & $0.03-0.15$ & $*<0.01$ \\
\hline Others & 71 & 24 & 0.11 & $0.05-0.25$ & $*<0.01$ \\
\hline \multicolumn{6}{|l|}{ Work place } \\
\hline University hospital & 83 & 14 & 1 & ref & ref \\
\hline Polyclinic hospital & 167 & 24 & 1.15 & $0.54-2.45$ & 0.72 \\
\hline Hospital & 221 & 15 & 2.03 & $0.89-4.66$ & 0.09 \\
\hline Clinic & 256 & 17 & 1.55 & $0.66-3.62$ & 0.31 \\
\hline Others & 31 & II & 0.69 & $0.25-1.92$ & 0.48 \\
\hline Exercise volume $^{\mathrm{a}}$ & N/A & N/A & 1.04 & $0.98-1.09$ & 0.15 \\
\hline \multicolumn{6}{|l|}{ Hyperlipidemia } \\
\hline \multicolumn{6}{|l|}{ Age } \\
\hline 24-30 & 56 & 15 & I & ref & ref \\
\hline $30-40$ & 210 & 49 & 0.88 & $0.57-2.30$ & 0.71 \\
\hline $40-50$ & 202 & 63 & 0.93 & $0.44-1.76$ & 0.72 \\
\hline $50-60$ & $|8|$ & 49 & 0.69 & $0.45-1.90$ & 0.84 \\
\hline$\geq 60$ & 10 & 4 & 0.69 & $0.17-2.80$ & 0.6 \\
\hline \multicolumn{6}{|l|}{ Specialty } \\
\hline Internal medicine & 336 & 51 & I & ref & ref \\
\hline Surgery & 97 & 61 & 0.26 & $0.16-0.4 \mid$ & $*<0.01$ \\
\hline General medicine & 139 & 16 & 1.16 & $0.63-2.13$ & 0.64 \\
\hline Pediatrics & 27 & 17 & 0.23 & $0.12-0.46$ & $*<0.01$ \\
\hline Others & 60 & 35 & 0.29 & $0.17-0.5 \mid$ & $*<0.01$ \\
\hline \multicolumn{6}{|l|}{ Work place } \\
\hline University hospital & 73 & 24 & 1 & ref & ref \\
\hline Polyclinic hospital & 133 & 58 & 0.75 & $0.4 I-1.35$ & 0.33 \\
\hline Hospital & 191 & 45 & 1.13 & $0.62-2.06$ & 0.69 \\
\hline Clinic & 234 & 39 & 1.21 & $0.65-2.27$ & 0.55 \\
\hline Others & 28 & 14 & $0.7 \mathrm{I}$ & $0.30-1.68$ & 0.44 \\
\hline Exercise volume ${ }^{a}$ & N/A & N/A & 1.02 & $1.02-10.9$ & $*<0.01$ \\
\hline \multicolumn{6}{|l|}{ Apoplexia } \\
\hline \multicolumn{6}{|l|}{ Age } \\
\hline 24-30 & 21 & 50 & I & ref & ref \\
\hline $30-40$ & 95 & 164 & 1.46 & $0.82-2.63$ & 0.2 \\
\hline $40-50$ & 102 & 163 & 1.66 & $0.92-2.99$ & 0.09 \\
\hline $50-60$ & 100 & 130 & 1.93 & $|.06-3.5|$ & 0.03 \\
\hline$\geq 60$ & 7 & 7 & 2.45 & $0.75-8.05$ & 0.14 \\
\hline \multicolumn{6}{|l|}{ Specialty } \\
\hline Internal medicine & 167 & 220 & I & ref & ref \\
\hline Surgery & 57 & 101 & 0.76 & $0.5 \mathrm{I}-\mathrm{I} .13$ & 0.17 \\
\hline General medicine & 69 & 86 & 1.09 & $0.74-1.6 \mid$ & 0.66 \\
\hline Pediatrics & 3 & 41 & 0.09 & $0.03-0.31$ & $*<0.01$ \\
\hline Others & 29 & 66 & 0.66 & $0.40-1.10$ & 0.11 \\
\hline
\end{tabular}


Table 2 (Continued)

\begin{tabular}{|c|c|c|c|c|c|}
\hline & \multicolumn{2}{|c|}{ Exercise recommendation } & \multirow[t]{2}{*}{ OR } & \multirow[t]{2}{*}{$95 \% \mathrm{Cl}$} & \multirow[t]{2}{*}{$P$-value } \\
\hline & $(+)$ & $(-)$ & & & \\
\hline \multicolumn{6}{|l|}{ Work place } \\
\hline University hospital & 36 & 61 & I & ref & ref \\
\hline Polyclinic hospital & 60 & $|3|$ & 0.8 & $0.47-1.34$ & 0.39 \\
\hline Hospital & 104 & 132 & 1.3 & $0.79-2.15$ & 0.31 \\
\hline Clinic & 113 & 160 & 1.04 & $0.63-1.7 \mid$ & 0.89 \\
\hline Others & 12 & 30 & 0.68 & $0.30-1.04$ & 0.35 \\
\hline Exercise volume ${ }^{\mathrm{a}}$ & $\mathrm{N} / \mathrm{A}$ & $\mathrm{N} / \mathrm{A}$ & 1.01 & $0.98-1.04$ & 0.45 \\
\hline \multicolumn{6}{|l|}{ Heart failure } \\
\hline \multicolumn{6}{|l|}{ Age } \\
\hline $24-30$ & 9 & 62 & I & ref & ref \\
\hline $30-40$ & 48 & 211 & 1.64 & $0.75-3.60$ & 0.22 \\
\hline $40-50$ & 48 & 217 & 1.61 & $0.73-3.55$ & 0.24 \\
\hline $50-60$ & 42 & 188 & 1.51 & $0.68-3.39$ & 0.31 \\
\hline$\geq 60$ & 3 & 11 & 2.11 & $0.46-9.62$ & 0.34 \\
\hline \multicolumn{6}{|l|}{ Specialty } \\
\hline Internal medicine & 95 & 292 & I & ref & ref \\
\hline Surgery & 11 & 147 & 0.23 & $0.12-0.45$ & $*<0.0$ I \\
\hline General medicine & 33 & 122 & 0.8 & $0.50-1.27$ & 0.34 \\
\hline Pediatrics & 2 & 42 & 0.14 & $0.03-0.60$ & $*<0.01$ \\
\hline Others & 9 & 86 & 0.34 & $0.16-0.73$ & $*<0.0$ I \\
\hline \multicolumn{6}{|l|}{ Work place } \\
\hline University hospital & 15 & 82 & I & ref & ref \\
\hline Polyclinic hospital & 25 & 166 & 0.85 & $0.42-1.75$ & 0.67 \\
\hline Hospital & 48 & 188 & 1.22 & $0.63-2.35$ & 0.56 \\
\hline Clinic & 57 & 216 & 1.01 & $0.53-1.95$ & 0.97 \\
\hline Others & 5 & 37 & 0.75 & $0.24-2.31$ & 0.61 \\
\hline Exercise volume ${ }^{\mathrm{a}}$ & $N / A$ & $\mathrm{~N} / \mathrm{A}$ & 1.04 & $1.01-1.07$ & $*<0.0$ I \\
\hline \multicolumn{6}{|l|}{ Hypertension } \\
\hline \multicolumn{6}{|l|}{ Age } \\
\hline $24-30$ & 39 & 32 & I & ref & ref \\
\hline $30-40$ & 170 & 89 & 1.6 & $0.90-2.82$ & 0.11 \\
\hline $40-50$ & 154 & 111 & 1.12 & $0.63-1.97$ & 0.71 \\
\hline $50-60$ & 144 & 86 & 1.23 & $0.69-2.21$ & 0.49 \\
\hline$\geq 60$ & 10 & 4 & 2.09 & $0.55-7.91$ & 0.28 \\
\hline \multicolumn{6}{|l|}{ Specialty } \\
\hline Internal medicine & 274 & 113 & 1 & ref & ref \\
\hline Surgery & 69 & 89 & 0.34 & $0.23-0.52$ & $*<0.0$ I \\
\hline General medicine & 114 & 41 & 0.99 & $0.64-1.52$ & 0.95 \\
\hline Pediatrics & 16 & 28 & 0.22 & $0.12-0.44$ & $*<0.01$ \\
\hline Others & 44 & 51 & 0.37 & $0.22-0.61$ & $*<0.0$ I \\
\hline \multicolumn{6}{|l|}{ Work place } \\
\hline University hospital & 53 & 44 & I & ref & ref \\
\hline Polyclinic hospital & 101 & 90 & 0.95 & $0.57-1.59$ & 0.84 \\
\hline Hospital & 138 & 98 & 1.01 & $0.6 I-1.68$ & 0.96 \\
\hline Clinic & 201 & 72 & 1.67 & $1.00-2.81$ & 0.05 \\
\hline Others & 24 & 18 & 1.25 & $0.57-2.75$ & 0.58 \\
\hline Exercise volume $\mathrm{e}^{\mathrm{a}}$ & N/A & $\mathrm{N} / \mathrm{A}$ & 1.05 & $1.02-1.08$ & $*<0.01$ \\
\hline
\end{tabular}

Notes: ${ }^{\mathrm{a}}$ The relationship between exercise recommendation and their own exercise volume. $* \mathrm{P}<0.0 \mathrm{I}$.

Abbreviations: $\mathrm{Cl}$, confidence interval; ref, reference; N/A, not available; OR, odds ratio.

that primary care physicians whose specialty was surgery counseled less about lifestyle factors including exercise than those with a nonsurgical specialty, even after controlling for differences in health related attitudes and personal habits. Recently, Hung et al reported that nonsmoking physicians were more likely to perform lifestyle intervention, and those who exercised at least one day per week were more likely to recommend limiting alcohol use in their patients with hypertension. ${ }^{11}$ In the present study, we found the primary care physicians whose specialties were internal medicine and 
general medicine highly recommended exercise compared with those whose specialties were surgery and pediatrics in cases of diabetes mellitus, hyperlipidemia, heart failure, and arterial hypertension. In addition, the primary care physicians whose specialty was pediatrics recommended exercise less often in cases of apoplexia. In addition, the primary care physicians' own exercise habits were positively associated with their recommendation of exercise for patients with hyperlipidemia, heart failure, and hypertension. These results are consistent with previous studies. ${ }^{7,9,11}$ In the present study, primary care physicians less often recommended exercise in cases of apoplexia, heart failure, and arterial hypertension than in diabetic mellitus and hyperlipidemia. These results may show that primary care physicians have difficulty providing exercise counseling and exercise prescription for cases of heart failure, apoplexia, and arterial hypertension because these diseases have large variations from a mild stage to a severe stage. Further studies will need to clarify why primary care physicians recommended exercise less often for cases of apoplexia, heart failure, and arterial hypertension than for diabetic mellitus and hyperlipidemia. Since there were several differences in primary care physicians' exercise counseling for cases of cardiovascular disease and metabolic syndrome in terms of their specialty and own exercise habits in the present study, it was suggested that there should be greater recognition and implementation of exercise guidelines for cases of metabolic syndromes and cardiovascular diseases by primary care physicians, regardless of their specialty and own exercise habits.

There are several limitations in this study. First, since the study instrument was a mailed questionnaire, there may have been self-selection bias. Second, it should be noted that the results of this study were not obtained by objective evaluation of the actual exercise counseling performed by primary care physicians. Further study will need to investigate actual exercise counseling, such as counseling time and detailed content, by primary care physicians. Third, the exercise volume of primary care physicians in this study did not reach the levels recommended in several sets of guidelines, as we previously reported. ${ }^{13}$ The low exercise volume of primary care physicians in the present study may thus have affected the results.

In conclusion, the exercise recommendation level of primary care physicians in this study was high for cases of metabolic syndrome, but low for cardiovascular diseases. The primary care physicians whose specialties were internal medicine and general medicine significantly more often recommended exercise in cases of diabetes mellitus, hyperlipidemia, heart failure, and hypertension. In addition, their own exercise habits were positively associated with their recommendation of exercise in cases of hyperlipidemia, heart failure, and hypertension.

\section{Acknowledgments}

We thank Minami Watanabe, Yuko Suda, Yukari Hoshino, and Aiko Oashi for their excellent assistance.

\section{Disclosure}

The authors declare no conflicts of interest.

\section{References}

1. Blair SN, Kampert JB, Kohl HW 3rd, et al. Influences of cardiorespiratory fitness and other precursors on cardiovascular disease and all-cause mortality in men and women. JAMA. 1996;276(3):205-210.

2. Underwood FB, Laughlin MH, Sturek M. Altered control of calcium in coronary smooth muscle cells by exercise training. Med Sci Sports Exerc. 1994;26(10):1230-1238.

3. Kriska AM, Blair SN, Pereira MA. The potential role of physical activity in the prevention of non-insulin-dependent diabetes mellitus: the epidemiological evidence. Exerc Sport Sci Rev. 1994;22:121-143.

4. Boyden TW, Pamenter RW, Going SB, et al. Resistance exercise training is associated with decreases in serum low-density lipoprotein cholesterol levels in premenopausal women. Arch Intern Med. 1993;153(1):97-100.

5. Goldberg L, Elliot DL. The effect of physical activity on lipid and lipoprotein levels. Med Clin North Am. 1985;69(1):41-55.

6. Clyne $\mathrm{N}$. The importance of exercise training in predialysis patients with chronic kidney disease. Clin Nephrol. 2004;61 Suppl 1:S10-S13.

7. Wells KB, Lewis CE, Leake B, Ware JE. Do physicians preach what they practice? A study of physicians' health habits and counseling practices. JAMA.1984;252(20):2846-2848.

8. Sherman SE, Hershman WY. Exercise counseling: how do general internists do? J Gen Intern Med. 1993;8(5):243-248.

9. Abramson S, Stein J, Schaufele M, Frates E, Rogan S. Personal exercise habits and counseling practices of primary care physicians: a national survey. Clin J Sport Med. 2000;10(1):40-48.

10. Kawakami M, Nakamura S, Fumimoto H, Takizawa J, Baba M. Relation between smoking status of physicians and their enthusiasm to offer smoking cessation advice. Intern Med. 1997;36(3):162-165.

11. Hung OY, Keenan NL, Fang J. Physicians' health habits are associated with lifestyle counseling for hypertensive patients. Am J Hypertens. 2013;26(2):201-208.

12. Morishita Y, Numata A, Miki A, et al. Primary care physicians' own exercise habits influence exercise counseling for patients with chronic kidney disease: a cross-sectional study. BMC Nephrol. 2014;15(1):48.

13. Morishita Y, Miki A, Okada M, et al. Association of primary care physicians' exercise habits and their age, specialty, and workplace. J Multidiscip Healthc. 2013;6:409-414.

14. Godin G, Shephard RJ. An evaluation of the potential role of the physician in influencing community exercise behavior. Am J Health Promot. 1990;4(4):255-259.

15. Wallace PG, Haines AP. General practitioner and health promotion: what patients think. Br Med J (Clin Res Ed). 1984;289(6444): 534-536.

16. Logsdon DN, Lazaro CM, Meier RV. The feasibility of behavioral risk reduction in primary medical care. Am J Prev Med. 1989;5(5):249-256. 
International Journal of General Medicine

Dovepress

\section{Publish your work in this journal}

The International Journal of General Medicine is an international, peer-reviewed open-access journal that focuses on general and internal medicine, pathogenesis, epidemiology, diagnosis, monitoring and treatment protocols. The journal is characterized by the rapid reporting of reviews, original research and clinical studies across all disease areas.
A key focus is the elucidation of disease processes and management protocols resulting in improved outcomes for the patient. The manuscript management system is completely online and includes a very quick and fair peer-review system. Visit http://www.dovepress.com/ testimonials.php to read real quotes from published authors.

Submit your manuscript here: http://www.dovepress.com/international-journal-of-general-medicine-journal 